\title{
A few results on generalized Janowski type functions associated with $(j, k)$-symmetrical functions
}

F. S. M. Al Sarari

Department of Studies in Mathematics,

University of Mysore, India

email: alsrary@yahoo.com

T. Al-Hawary

Department of Applied Science, Ajloun College, Al-Balqa Applied

University, Ajloun, Jordan email: tariq_amh@yahoo.com

\section{B. A. Frasin}

Department of Mathematics,

Faculty of Science, Al al-Bayt University, Jordan email: bafrasin@yahoo.com

\section{S. Latha}

Department of Mathematics, Yuvaraja's College, University of Mysore, India email: drlatha@gmail.com

\begin{abstract}
The aim of the present article is to introduce and study new subclass of Janowski type functions defined using notions of Janowski functions and $(j, k)$-symmetrical functions. Certain interesting coefficient inequalities, sufficiency criteria, distortion theorem, neighborhood property are investigated for this class.
\end{abstract}

\section{Introduction and definitions}

Let $\mathcal{A}$ denote the class of functions of form

$$
f(z)=z+\sum_{n=2}^{\infty} a_{n} z^{n}
$$

2010 Mathematics Subject Classification: 30C45

Key words and phrases: analytic functions, $\alpha$-starlike, $\alpha$-close-to-convex, differential subordination, $(j, k)$-symmetric points 
which are analytic in the open unit $\operatorname{disk} \mathcal{U}=\{z: z \in \mathbb{C}$ and $|z|<1\}$, and $\mathcal{S}$ denote the subclass of $\mathcal{A}$ consisting of all function which are univalent in $\mathcal{U}$. For any $\mathrm{f} \in \mathcal{A}$, $\rho$-neighborhood of $\mathrm{f}(z)$ can be defined as:

$$
\mathcal{N}_{\rho}(f)=\left\{g \in \mathcal{A}: g(z)=z+\sum_{n=2}^{\infty} b_{n} z^{n}, \quad \sum_{n=2}^{\infty} n\left|a_{n}-b_{n}\right| \leq \rho\right\} .
$$

For $e(z)=z$, we can see that

$$
\mathcal{N}_{\rho}(e)=\left\{g \in \mathcal{A}: g(z)=z+\sum_{n=2}^{\infty} b_{n} z^{n}, \quad \sum_{n=2}^{\infty} n\left|b_{n}\right| \leq \rho\right\} .
$$

The idea of neighborhoods was first introduced by Goodman [9] which was further generalized by Ruscheweyh [5]. For $\mathrm{f}$ and $\mathrm{g}$ be analytic in $\mathcal{U}$, we say that the function $\mathrm{f}$ is subordinate to $\mathrm{g}$ in $\mathcal{U}$, if there exists an analytic function $\omega$ in $\mathcal{U}$ such that $|\omega(z)|<1$ and $f(z)=g(\omega(z))$, and we denote this by $f \prec g$. If $g$ is univalent in $\mathcal{U}$, then the subordination is equivalent to $f(0)=g(0)$ and $f(\mathcal{U}) \subset \mathrm{g}(\mathcal{U})$.

Using the principle of the subordination we define the class $\mathcal{P}$ of functions with positive real parts.

Definition 1 [6] Let $\mathcal{P}$ denote the class of analytic functions of the form $\mathrm{p}(z)=1+\sum_{n=1}^{\infty} \mathrm{p}_{\mathrm{n}} z^{\mathrm{n}}$ defined on $\mathcal{U}$ and satisfying $\mathrm{p}(0)=1, \mathfrak{R}\{\mathrm{p}(z)\}>0, z \in$ $\mathcal{U}$.

Any function $p$ in $\mathcal{P}$ has the representation $p(z)=\frac{1+w(z)}{1-w(z)}$ where $w \in \Omega$ and

$$
\Omega=\{w \in \mathcal{A} \text { and } w(0)=0|w(z)|<1\} .
$$

The class of functions $\mathcal{P}$ with positive real part plays a crucial role in geometric function theory. Its significance can be seen from the fact that simple subclasses like class of starlike $\mathcal{S}^{*}$, class of convex functions $\mathcal{C}$, class of starlike functions with respect to symmetric points $\mathcal{S}_{\mathrm{s}}^{*}$ have been defined by using the concept of class of functions with positive real part.

Definition 2 [1] Let $\mathcal{P}[\mathrm{A}, \mathrm{B}]$, where $-1 \leq \mathrm{B}<\mathrm{A} \leq 1$, denote the class of analytic function $\mathrm{p}$ defined on $\mathcal{U}$ with the representation $\mathrm{p}(z)=\frac{1+\mathrm{A} w(z)}{1+\mathrm{B} w(z)}, \quad z \in$ $\mathcal{U}, w \in \Omega . p \in \mathcal{P}[A, B]$ if and only if $p(z) \prec \frac{1+A z}{1+B z}$. 
Definition 3 Let $\mathrm{k}$ be a positive integer. A domain $\mathcal{D}$ is said to be $\mathrm{k}$-fold symmetric if a rotation of $\mathcal{D}$ about the origin through an angle $\frac{2 \pi}{\mathrm{k}}$ carries $\mathcal{D}$ onto itself. A function $\mathrm{f}$ is said to be $\mathrm{k}$-fold symmetric in $\mathcal{U}$ if for every $z$ in $\mathcal{U}$

$$
f\left(e^{\frac{2 \pi i}{k}} z\right)=e^{\frac{2 \pi i}{k}} f(z) .
$$

The family of all k-fold symmetric functions is denoted by $\mathcal{S}^{\mathrm{k}}$ and for $\mathrm{k}=2$ we get class of odd univalent functions.

The notion of $(j, k)$-symmetrical functions $(j=0,1,2, \ldots, k-1 ; k=2,3, \ldots)$ is a generalization of the notion of even, odd, k-symmetrical functions and also generalize the well-known result that each function defined on a symmetrical subset can be uniquely expressed as the sum of an even function and an odd function.

The theory of $(j, k)$ symmetrical functions has many interesting applications, for instance in the investigation of the set of fixed points of mappings, for the estimation of the absolute value of some integrals, and for obtaining some results of the type of Cartan's uniqueness theorem for holomorphic mappings [10].

Definition 4 Let $\varepsilon=\left(e^{\frac{2 \pi i}{k}}\right)$ and $j=0,1,2, \ldots, k-1$ where $\mathrm{k} \geq 2$ is a natural number. A function $\mathrm{f}: \mathcal{D} \mapsto \mathbb{C}$ where $\mathcal{D}$ is a $\mathrm{k}$-fold symmetric set, is called $(\mathrm{j}, \mathrm{k})$-symmetrical if

$$
f(\varepsilon z)=\varepsilon^{j} f(z), \quad z \in \mathcal{U} .
$$

We note that the family of all $(\mathfrak{j}, \mathrm{k})$-symmetric functions is denoted be $\mathcal{S}^{(j, k)}$. Also, $\mathcal{S}^{(0,2)}, \mathcal{S}^{(1,2)}$ and $\mathcal{S}^{(1, k)}$ the classes of even, odd and k-symmetric functions respectively. We have the following decomposition theorem.

Theorem 1 [10] For every mapping $\mathrm{f}: \mathcal{D} \mapsto \mathbb{C}$, where $\mathcal{D}$ is a $\mathrm{k}$-fold symmetric set, there exists exactly the sequence of $(\mathfrak{j}, \mathrm{k})$ - symmetrical functions $\mathrm{f}_{\mathfrak{j}, \mathrm{k}}$,

$$
f(z)=\sum_{j=0}^{k-1} f_{j, k}(z)
$$

where

$$
\begin{gathered}
f_{j, k}(z)=\frac{1}{k} \sum_{v=0}^{k-1} \varepsilon^{-v j} f\left(\varepsilon^{v} z\right), \\
(f \in \mathcal{A} ; k=1,2, \ldots ; j=0,1,2, \ldots, k-1)
\end{gathered}
$$


From (4) we can get

$$
\mathrm{f}_{j, k}(z)=\frac{1}{k} \sum_{v=0}^{k-1} \varepsilon^{-v j} f\left(\varepsilon^{v} z\right)=\frac{1}{k} \sum_{v=0}^{k-1} \varepsilon^{-v j}\left(\sum_{n=1}^{\infty} a_{n}\left(\varepsilon^{v} z\right)^{n}\right),
$$

then

$$
f_{j, k}(z)=\sum_{n=1}^{\infty} \delta_{n, j} a_{n} z^{n}, \quad a_{1}=1, \quad \delta_{n, j}=\frac{1}{k} \sum_{v=0}^{k-1} \varepsilon^{(n-j) v}= \begin{cases}1, & n=l k+j ; \\ 0, & n \neq l k+j .\end{cases}
$$

Alsarari and Latha [4] introduced and studied the classes $\mathcal{S}^{(j, k)}(A, B)$ and $\mathcal{K}^{(j, k)}(A, B)$ which are starlike and convex with respect to $(j, k)$-symmetric points, respectively.

Definition 5 [4] A function $\mathrm{f}$ in $\mathcal{A}$ is said to belong to the class $\mathcal{S}^{(\mathrm{j}, \mathrm{k})}(\mathrm{A}, \mathrm{B})$, $(-1 \leq \mathrm{B}<\mathrm{A} \leq 1)$ if

$$
\frac{z f^{\prime}(z)}{f_{j, k}(z)} \prec \frac{1+A z}{1+B z}, \quad z \in \mathcal{U},
$$

where $\mathrm{f}_{\mathrm{j}, \mathrm{k}}(\boldsymbol{z})$ defined by $(5)$.

This class is generalizes the classes studied by Ohsang and Yaungjae [7] and Sakaguchi [8].

We need the following lemma to prove our main results.

Lemma 1 [3] Let $p(z)=1+\sum_{n=1}^{\infty} p_{n} z^{n} \in \mathcal{P}[A, B]$, then for $n \geq 1$,

$$
\left|p_{n}\right| \leq(A-B) \text {. }
$$

\section{Main results}

Theorem 2 If $\mathrm{f} \in \mathcal{S}^{(\mathrm{j}, \mathrm{k})}(\mathrm{A}, \mathrm{B})$, then for $\mathrm{n} \geq 2,-1 \leq \mathrm{B}<\mathrm{A} \leq 1$.

$$
\left|a_{n}\right| \leq \prod_{m=1}^{n-1} \frac{\delta_{m, j}[(A-B)-1]+m}{m+1-\delta_{m+1, j}}
$$

where $\delta_{n, j}$ by (5). 
Proof. By Definition (5) we have

$$
\frac{z f^{\prime}(z)}{f_{j, k}(z)}=p(z), \quad p \in \mathcal{P}[A, B],
$$

then we have

$$
z f^{\prime}(z)=\left[1+\sum_{n=1}^{\infty} p_{n} z^{\mathfrak{n}}\right] f_{j, k}(z)
$$

by (1) and (5), we have

$$
\left(1-\delta_{1, j}\right) z+\sum_{n=2}^{\infty}\left[n-\delta_{n, j}\right] a_{n} z^{n}=\left[\sum_{n=1}^{\infty} p_{n} z^{n}\right]\left[\sum_{n=1}^{\infty} \delta_{n, j} a_{n} z^{n}\right] .
$$

Equating coefficients of $z^{\mathfrak{n}}$ on both sides, we have

$$
a_{n}=\frac{1}{\left[n-\delta_{n, j}\right]} \sum_{m=1}^{n-1} p_{m} \delta_{n-m, j} a_{n-m}, \quad \delta_{1, j}=1,
$$

by Lemma 1, we have

$$
\left|a_{n}\right| \leq \frac{(A-B)}{\left[n-\delta_{n, j}\right]} \sum_{m=1}^{n-1} \delta_{m, j}\left|a_{m}\right| .
$$

Now we want to prove that

$$
\frac{(A-B)}{\left[n-\delta_{n, j}\right]} \sum_{m=1}^{n-1} \delta_{m, j}\left|a_{m}\right| \leq \prod_{m=1}^{n-1} \frac{\delta_{m, j}[(A-B)-1]+m}{\left[m+1-\delta_{m+1, j}\right]} .
$$

For this, we use the induction method. The result is true for $n=2$ and 3 . Let the hypothesis be true for $n=m$, we have

$$
\frac{(A-B)}{\left[m-\delta_{m, j}\right]} \sum_{r=1}^{m-1} \delta_{r, j}\left|a_{r}\right| \leq \prod_{r=1}^{m-1} \frac{\delta_{r, j}[(A-B)-1]+r}{\left[r+1-\delta_{r+1, j}\right]} .
$$

Multiplying both sides by $\frac{\delta_{\mathfrak{m} . j}[(A-B)-1]+m}{\left[m+1-\delta_{m+1, j}\right]}$, we get

$$
\prod_{r=1}^{m} \frac{\delta_{r, j}[(A-B)-1]+r}{\left[r+1-\delta_{r+1, j}\right]} \geq \frac{\delta_{m . j}[(A-B)-1]+m}{\left[m+1-\delta_{m+1, j}\right]} \cdot \frac{(A-B)}{\left[m-\delta_{m, j}\right]} \sum_{r=1}^{m-1} \delta_{r, j}\left|a_{r}\right|,
$$


since

$$
\begin{aligned}
& \frac{\delta_{m . j}[(A-B)-1]+m}{\left[m+1-\delta_{m+1, j}\right]} \cdot \frac{(A-B)}{\left[m-\delta_{m, j}\right]} \sum_{r=1}^{m-1} \delta_{r, j}\left|a_{r}\right| \\
= & \frac{(A-B)}{\left[m+1-\delta_{m+1, j}\right]} \cdot\left[1+\frac{\delta_{m, j}(A-B)}{\left[m-\delta_{m, j}\right]}\right] \sum_{r=1}^{m-1} \delta_{r, j}\left|a_{r}\right|, \\
\geq & \frac{(A-B)}{\left[m+1-\delta_{m+1, j}\right]} \cdot\left[\sum_{r=1}^{m-1} \delta_{r, j}\left|a_{r}\right|+\delta_{m, j}\left|a_{m}\right|\right], \\
= & \frac{(A-B)}{\left[m+1-\delta_{m+1, j}\right]} \cdot\left[\sum_{r=1}^{m} \delta_{r, j}\left|a_{r}\right|\right] .
\end{aligned}
$$

That is

$$
\left|a_{m+1}\right| \leq \frac{(A-B)}{\left[m-\delta_{m, j}\right]} \sum_{r=1}^{m} \delta_{r, j}\left|a_{r}\right| \leq \prod_{r=1}^{m} \frac{\delta_{r, j}[(A-B)-1]+r}{\left[r+1-\delta_{r+1, j}\right]},
$$

which shows that inequality (6) is true for $n=m+1$. This completes the proof.

Theorem 3 Let $\mathrm{f}(z)=z+\sum_{n=2}^{\infty} a_{n} z^{n}$, be analytic in $\mathcal{U}$, for $(-1 \leq \mathrm{B}<\mathrm{A} \leq$ 1), we have

$$
\sum_{n=2}^{\infty}\left\{\left(n-\delta_{n, j}\right)+\left|A \delta_{n, j}-B n\right|\right\}\left|a_{n}\right| \leq(A-B) .
$$

Then, $\mathrm{f}(z) \in \mathcal{S}^{(\mathrm{j}, \mathrm{k})}(\mathrm{A}, \mathrm{B})$.

Proof. For the proof of Theorem 3, it suffices to show that the values for $\frac{z f^{\prime}(z)}{f_{j, k}(z)}$, satisfy

$$
\left|\frac{z f^{\prime}(z)-f_{j, k}(z)}{A f_{j, k}(z)-B z f^{\prime}(z)}\right| \leq 1
$$

we have 


$$
\begin{aligned}
\left|\frac{z f^{\prime}(z)-f_{j, k}(z)}{A f_{j, k}(z)-B z f^{\prime}(z)}\right| & =\left|\frac{\sum_{n=2}^{\infty}\left(n-\delta_{n, j}\right) a_{n} z^{n-1}}{(A-B)+\sum_{n=2}^{\infty}\left\{A \delta_{n, j}-B n\right\} a_{n} z^{n-1}}\right| \\
& \leq \frac{\sum_{n=2}^{\infty}\left(n-\delta_{n, j}\right)\left|a_{n}\right||z|^{n-1}}{(A-B)-\sum_{n=2}^{\infty}\left|A \delta_{n, j}-B n\right|\left|a_{n}\right||z|^{n-1}} \\
& \leq \frac{\sum_{n=2}^{\infty}\left(n-\delta_{n, j}\right)\left|a_{n}\right|}{(A-B)-\sum_{n=2}^{\infty}\left|A \delta_{n, j}-B n\right|\left|a_{n}\right|} .
\end{aligned}
$$

This last expression is bounded above by 1 if

$$
\sum_{n=2}^{\infty}\left\{\left(n-\delta_{n, j}\right)+\left|A \delta_{n, j}-B n\right|\right\}\left|a_{n}\right| \leq(A-B),
$$

hence $\left|\frac{z f^{\prime}(z)-f_{j, k}(z)}{A f_{j, k}(z)-B z f^{\prime}(z)}\right| \leq 1$, and Theorem 3 is proved.

Theorem 4 Let $\mathrm{f}(z) \in \mathcal{S}^{(\mathrm{j}, \mathrm{k})}(\mathrm{A}, \mathrm{B})$, for $(-1<\mathrm{B}<\mathrm{A} \leq 1)$, then

$$
|z|-\sum_{n=2}^{i}\left|a_{n}\right||z|^{n}-\tau_{i}|z|^{i+1} \leq|f(z)| \leq|z|+\sum_{n=2}^{i}\left|a_{n}\right||z|^{n}+\tau_{i}|z|^{i+1},
$$

where

$$
\tau_{i}=\frac{(A-B)-\sum_{n=2}^{i}\left\{\left(n-\delta_{n, j}\right)+\left|A \delta_{n, j}-B n\right|\right\}\left|a_{n}\right|}{\left\{(i+1)(1-|B|)-[1-|A|] \delta_{i+1, j}\right\}} .
$$

Proof. From Theorem 3 we have

$$
\begin{aligned}
& \sum_{n=i+1}^{\infty}\left\{\left(n-\delta_{n, j}\right)+\left|A \delta_{n, j}-B n\right|\right\}\left|a_{n}\right| \\
& \quad \leq(A-B)-\sum_{n=2}^{i}\left\{\left(n-\delta_{n, j}\right)+\left|A \delta_{n, j}-B n\right|\right\}\left|a_{n}\right| .
\end{aligned}
$$

On the other hand

$$
\left(n-\delta_{n, j}\right)+\left|A \delta_{n, j}-B n\right| \geq n(1-|B|)-[1-|A|] \delta_{n, j}
$$

and hence $n(1-|B|)-[1-|A|] \delta_{n, j}$ is monotonically increasing with respect to n. So we can write

$$
\left\{(i+1)(1-|B|)-[1-|A|] \delta_{i+1, j}\right\} \sum_{n=i+1}^{\infty}\left|a_{n}\right|
$$




$$
\leq(A-B)-\sum_{n=2}^{i}\left\{\left(n-\delta_{n, j}\right)+\left|A \delta_{n, j}-B n\right|\right\}\left|a_{n}\right|
$$

which implies that

$$
\sum_{n=i+1}^{\infty}\left|a_{n}\right| \leq \tau_{i}
$$

hence we have

$$
|f(z)| \leq|z|+\sum_{n=2}^{i}\left|a_{n}\right||z|^{n}+\tau_{i}|z|^{i+1}
$$

and

$$
|f(z)| \geq|z|-\sum_{n=2}^{i}\left|a_{n}\right||z|^{n}-\tau_{i}|z|^{i+1} .
$$

This completes the proof of theorem.

Theorem 5 For $(-1<B<A \leq 1)$,

$$
\mathcal{S}^{(j, k)}(\mathrm{A}, \mathrm{B}) \subseteq \mathcal{N}_{\rho}(\mathrm{e}),
$$

where

$$
\rho=\left[\frac{(A-B)\left\{2(1-|B|)-(1-|A|) \delta_{2, j}+1\right\}}{2(1-|B|)-(1-|A|) \delta_{2, j}}\right] .
$$

Proof. For function $f \in \mathcal{S}^{(j, k)}(A, B)$, by Theorem 3, immediately yields

$$
\left\{2(1-|B|)-(1-|A|) \delta_{2, j}\right\} \sum_{n=2}^{\infty}\left|a_{n}\right| \leq(A-B),
$$

so, that

$$
\sum_{n=2}^{\infty}\left|a_{n}\right| \leq \frac{(A-B)}{2(1-|B|)-(1-|A|) \delta_{2, j}} .
$$

On the other hand, we also find from Theorem 3 ,

$$
\sum_{n=2}^{\infty}\left(n-\delta_{n, j}\right)\left|a_{n}\right| \leq(A-B),
$$

also

$$
\sum_{n=2}^{\infty}(n-1)\left|a_{n}\right| \leq(A-B)
$$


or

$$
\sum_{n=2}^{\infty} n\left|a_{n}\right| \leq(A-B)+\sum_{n=2}^{\infty}\left|a_{n}\right|
$$

that is,

$$
\sum_{n=2}^{\infty} n\left|a_{n}\right| \leq\left[\frac{(A-B)\left\{2(1-|B|)-(1-|A|) \delta_{2, j}+1\right\}}{2(1-|B|)-(1-|A|) \delta_{2, j}}\right]=\rho
$$

which, in view of the definition (3), proves Theorem 5.

Now we define the neighborhood for each of the class $\mathcal{S}^{(j, k)}(A, B)$.

A function $f \in \mathcal{A}$ is said to be in class $\mathcal{S}^{(j, k)}(A, B, \eta)$ if there exists $g \in$ $\mathcal{S}^{(j, k)}(A, B)$ such that

$$
\left|\frac{f(z)}{g(z)}-1\right|<1-\eta
$$

Theorem 6 Let $\mathrm{g} \in \mathcal{S}^{(\mathrm{j}, \mathrm{k})}(\mathrm{A}, \mathrm{B})$, and suppose that

$$
\eta=1-\frac{\rho\left\{2(1-|B|)-(1-|A|) \delta_{2, j}\right\}}{2\left\{2(1-|B|)-(1-|A|) \delta_{2, j}-(A-B)\right\}},
$$

then

$$
\mathcal{N}_{\rho}(g) \subseteq \mathcal{S}^{(\mathrm{j}, \mathrm{k})}(\mathrm{A}, \mathrm{B}, \eta)
$$

Proof. Suppose that $f \in \mathcal{N}_{\rho}(g)$. We then find from (2), that

$$
\sum_{n=2}^{\infty} n\left|a_{n}-b_{n}\right| \leq \rho
$$

which readily implies the coefficient inequality

$$
\sum_{n=2}^{\infty}\left|a_{n}-b_{n}\right| \leq \frac{\rho}{2}
$$

Next, since $g \in \mathcal{S}^{(j, k)}(A, B)$, from $(7)$, we have

$$
\sum_{n=2}^{\infty}\left|b_{n}\right| \leq \frac{(A-B)}{2(1-|B|)-(1-|A|) \delta_{2, j}}
$$


so that

$$
\begin{aligned}
\left|\frac{f(z)}{g(z)}-1\right| & \leq \frac{\sum_{n=2}^{\infty}\left|a_{n}-b_{n}\right|}{1-\sum_{n=2}^{\infty}\left|b_{n}\right|} \\
& \leq \frac{\rho\left\{2(1-|B|)-(1-|A|) \delta_{2, j}\right\}}{2\left\{2(1-|B|)-(1-|A|) \delta_{2, j} \mid-(A-B)\right\}} \\
& =1-\eta .
\end{aligned}
$$

That shows that $f \in \mathcal{S}^{(j, k)}(A, B, \alpha, \eta)$ for $\eta$ given by (8), which completes the proof.

\section{Acknowledgements}

The authors would like to thank the referee for his helpful comments and suggestions.

\section{References}

[1] W. Janowski, Some extremal problems for certain families of analytic functions, I. Ann. Polon. Math. 28 (1973), 297-326.

[2] A. Schild, On a class of functions schlicht in the unit circle, Proc. Amer. Math. Soc. 5 (1954), 115-120, MR 15, 694.

[3] M. K. Aouf, On a class of $p$-valent starlike functions of order $\alpha$, Internat. J. Math. Math. Sci. 1 (10) (1987), 733-744.

[4] F. Alsarari and S. Latha, A few results on functions that are Janowski starlike related to (j, k)-symmetric points, Octo. Math. Maga 21 (2), (October, 2013), 556-563.

[5] S. Ruschewyh, Neighborhoods of univalent functions, Proc. Amer. Math. Soc., 81 (1981), 521-527.

[6] P. L. Duren, Univalent Functions, Springer-Verlag, (1983).

[7] K. Ohsang, and S. Yaungjae, A certain subclass of Janowski type functions associated with k-symmetric points, Commun. Korean. Math. Soc. 28 (2013), 134-154.

[8] K. Sakaguchi, On a certain univalent mapping, J. Math. Soc. Japan, 11 (1959), 72-75. 
[9] A.W. Goodman, Univalent functions and nonanalytic curves, Proc. Amer. Math. Soc., 8 (1957), 598-601.

[10] P. Liczberski and J. Połubiński, On (j,k)-symmetrical functions, Math. Bohem. 120 (1) (1995), 13-28.

Received: March 4, 2016 